\title{
OGLE-2014-BLG-1112LB: A Microlensing Brown Dwarf Detected through the Channel of a Gravitational Binary-lens Event
}

\author{
C. $\operatorname{Han}^{1}$, A. Udalski ${ }^{2,11}$, V. Bozza ${ }^{3,4,12}$, \\ and \\ M. K. Szymański ${ }^{2}$, I. Soszyński ${ }^{2}$, J. Skowron ${ }^{2}$, P. Mróz ${ }^{2}$, R. Poleski ${ }^{2,5}$, P. Pietrukowicz ${ }^{2}$, , \\ S. Kozłowski ${ }^{2}$, K. Ulaczyk ${ }^{2}$, Ł. Wyrzykowski ${ }^{2}$ \\ (The OGLE Collaboration), \\ and \\ S. Calchi Novati ${ }^{3,6}$, G. D’Ago ${ }^{7}$, M. Dominik ${ }^{8}$, M. Hundertmark ${ }^{9}$, U. G. Jorgensen ${ }^{10}$, G. Scarpetta ${ }^{3,7}$ \\ (The MiNDSTEp Consortium) \\ ${ }^{1}$ Department of Physics, Chungbuk National University, Cheongju 28644, Korea \\ ${ }^{3}$ Dipartimento di Fisica "E. R. Caianiello,” Universitá di Salerno, Via Giovanni Paolo II, I-84084 Fisciano (SA), Italy \\ ${ }^{4}$ Istituto Nazionale di Fisica Nucleare, Sezione di Napoli, Via Cintia, I-80126 Napoli, Italy \\ ${ }^{5}$ Department of Astronomy, Ohio State University, 140 W. 18th Ave., Columbus, OH 43210, USA \\ 6 IPAC, Mail Code 100-22, Caltech, 1200 E. California Blvd., Pasadena, CA 91125, USA \\ 7 Istituto Internazionale per gli Alti Studi Scientifici (IIASS), Via G. Pellegrino 19, I-84019 Vietri sul Mare (SA), Italy \\ ${ }^{8}$ SUPA, School of Physics and Astronomy, University of St. Andrews, North Haugh, St. Andrews, KY16 9SS, UK \\ ${ }^{9}$ Institute and Centre for Star and Planet Formation, University of Copenhagen, Øster Voldgade 5, DK-1350 Copenhagen, Denmark \\ ${ }^{10}$ Astronomisches Rechen-Institut, Zentrum für Astronomie der Universität, Heidelberg, Mönchhofstr. 12-14, D-69120 Heidelberg, Germany \\ Received 2017 March 31; revised 2017 May 22; accepted 2017 May 29; published 2017 July 6
}

\begin{abstract}
Due to the nature of the gravitational field, microlensing, in principle, provides an important tool for detecting faint and even dark brown dwarfs. However, the number of identified brown dwarfs is limited due to the difficulty of the lens mass measurement that is needed to check the substellar nature of the lensing object. In this work, we report a microlensing brown dwarf discovered from an analysis of the gravitational binary-lens event OGLE-2014-BLG1112. We identify the brown dwarf nature of the lens companion by measuring the lens mass from the detections of both microlens-parallax and finite-source effects. We find that the companion has a mass of $(3.03 \pm 0.78) \times 10^{-2} M_{\odot}$ and it is orbiting a solar-type primary star with a mass of $1.07 \pm 0.28 M_{\odot}$. The estimated projected separation between the lens components is $9.63 \pm 1.33$ au and the distance to the lens is $4.84 \pm 0.67 \mathrm{kpc}$. We discuss the usefulness of space-based microlensing observations for detecting brown dwarfs through the channel of binary-lens events.
\end{abstract}

Key words: binaries: general - brown dwarfs - gravitational lensing: micro

\section{Introduction}

Considering that brown dwarfs are formed through a similar process to that of stars (Whitworth et al. 2007), it is suspected that the Galaxy should be teeming with brown dwarfs. Since the microlensing phenomenon occurs due to the gravitational field regardless of the brightness of lensing objects, it provides an important tool for detecting brown dwarfs, especially very faint and dark ones. Current microlensing surveys detect approximately 2000 lensing events each year and an important fraction of them might occur due to brown dwarfs.

However, the number of actually identified microlensing brown dwarfs is limited. Firm identification of a brown dwarf requires measuring the lens mass in order to check that the lens is a substellar object below the hydrogen-burning limit. For general lensing events, the only measurable quantity related to the mass of the lens is the Einstein timescale $t_{\mathrm{E}}$. However, the event timescale results from the combination of not only the lens mass but also the relative lens-source transverse speed and the distance to the lens. As a result, it is difficult to uniquely determine the lens mass based on only $t_{\mathrm{E}}$, which makes it

\footnotetext{
11 The OGLE Collaboration.

12 The MiNDSTEp Consortium.
}

difficult to identify the brown dwarf nature of lens objects for general lensing events. For the unique determination of the lens mass, one needs to additionally measure the angular Einstein radius $\theta_{\mathrm{E}}$ and the microlens parallax $\pi_{\mathrm{E}}$, which are related to the lens mass by the relation (Gould 2000)

$$
M=\frac{\theta_{\mathrm{E}}}{\kappa \pi_{\mathrm{E}}},
$$

where $\kappa=4 G /\left(c^{2} \mathrm{au}\right)$. One can measure the angular Einstein radius when a lensing event experiences finite-source effects. The microlens parallax can be measured by detecting subtle modulations in the lensing light curve produced by the change of the observer's position caused by the orbital motion of the Earth around the Sun. For general lensing events, unfortunately, the chance to measure both $\theta_{\mathrm{E}}$ and $\pi_{\mathrm{E}}$ is very low.

Although low for general lensing events, the chance of identifying a brown-dwarf lens is relatively high when events are produced by lenses composed of two masses. There are two major reasons for this. First, analysis of a binary-lens event yields additional information of the companion/primary mass ratio $q$ in addition to the timescale information of a single-mass event. Considering that the lenses of typical galactic microlensing events are low-mass stars, companions of binary lenses 
with $q<0.1$ are likely to be brown dwarfs (Shin et al. 2012). Second, the chance of identifying the brown dwarf nature of lens components by measuring both $\theta_{\mathrm{E}}$ and $\pi_{\mathrm{E}}$ is relatively high for a population of binary-lens events with long timescales. Most binary-lens events are identified from characteristic features related to caustics, which represent the positions on the source plane at which the lensing magnification of a point source becomes infinite. Due to the high magnification gradient around caustics, the light curve of a lensing event during the passage over or approach close to the caustics results in deviations caused by finite-source effects, enabling one to measure $\theta_{\mathrm{E}}$. Furthermore, timescales of binary-lens events tend to be longer than those of single-lens events and thus the chance of measuring $\pi_{\mathrm{E}}$ is also higher. Due to these reasons, 14 out of the total 16 microlensing brown dwarfs were detected through the channel of binary-lens events. See the list of microlensing brown dwarfs presented in Han et al. (2016).

Although it is useful, detecting brown dwarfs through the binary-lens event channel is still a difficult task. Similar to planets, brown dwarfs induce caustic-involved signals in lensing light curves. Since planet-induced caustics are usually very small, planetary lensing signals in most cases appear as characteristic short-term perturbations to the smooth and symmetric form of the light curve produced by the host of the planet (Mao \& Paczyński 1991; Gould 1992), making it easy to identify the planetary nature of the signal. On the other hand, the size of the caustic induced by a brown-dwarf companion can be considerable and thus brown-dwarf signals, in most cases, cannot be treated as perturbations. ${ }^{13}$ As a result, it is difficult to distinguish brown-dwarf binary-lens events from those produced by binaries with roughly equal masses (Gaudi 2003). This implies that finding brown-dwarf events requires detailed analyses of numerous binary-lens events, which comprise $\sim 10 \%$ of all events that are being detected with a rate of $\sim 2000 \mathrm{yr}^{-1}$. Due to the diversity and complexity of the light curves, which are described by many parameters, modeling the light curves of binary-lens events requires an intricate analysis procedure. Furthermore, describing caustic features in binary-lens light curves requires a numerical approach that demands considerable computing power. As a result, the difficulty of analyzing binary-lens events poses an important obstacle to finding brown dwarfs via microlensing.

In this paper, we report the microlensing discovery of a binary system that is composed of a brown dwarf and a solartype star. The low mass ratio between the lens components was found from analyses of anomalous events detected in the 2014 microlensing season. The angular Einstein radius is measured thanks to the good coverage of the caustic-approach region. The long timescale of the event, combined with extended baseline data, enable us to securely measure the microlens parallax. By measuring both $\theta_{\mathrm{E}}$ and $\pi_{\mathrm{E}}$, we uniquely determine the lens mass and identify the brown dwarf nature of the companion.

The paper is organized as follows. In Section 2, we describe observations of the microlensing event analyzed in this work and the data obtained from the observations. In Section 3, we explain the detailed analysis procedure used to interpret the

\footnotetext{
$\overline{13}$ We note that perturbations of light curves by binary lenses depend not only on the size of the caustic but also on the path and size of the source. Depending on the source trajectory with respect to the caustic and the size of the source star, the deviation caused by a low-mass binary companion can appear as a deviation of entire light curves (Ingrosso et al. 2009, 2011).
}

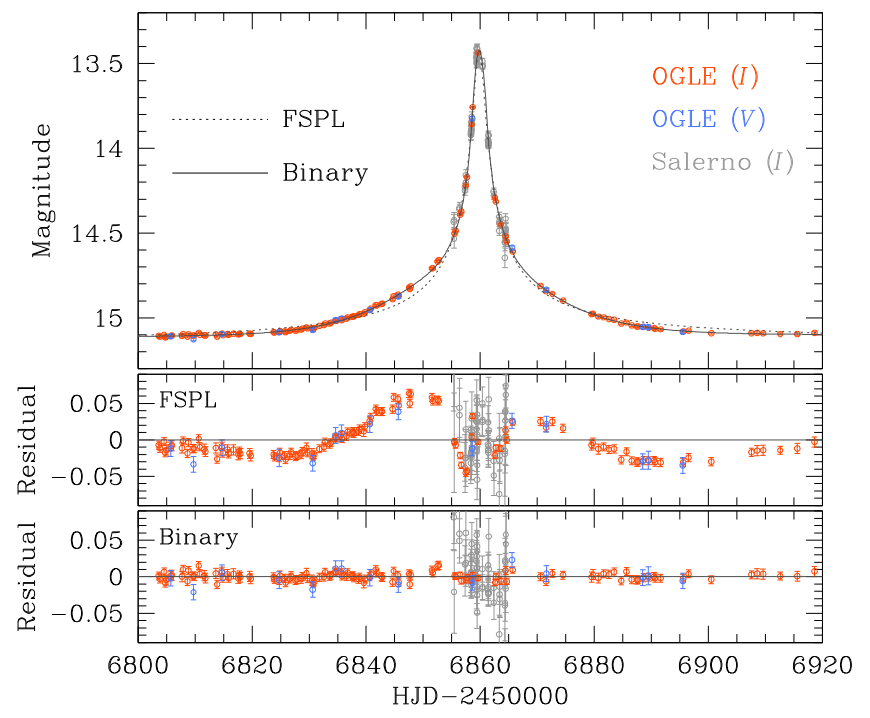

Figure 1. Light curve of the microlensing event OGLE-2014-BLG-1112. In the top panel, the solid curve superposed on the data points represents the best-fit binary-lens model, while the dotted curve is the finite-source point-lens (FSPL) model. The middle and bottom panels show the residual from the FSPL and binary-lens models, respectively.

observed data, and present the best-fit model of the lensing event. In Section 4, we present the physical parameters of the lens, including the mass and distance. In Section 5, we discuss the usefulness of space-based lensing observations for detecting microlensing brown dwarfs. We summarize the results and present our conclusions in Section 6.

\section{Data}

The brown dwarf was discovered from an analysis of the microlensing event OGLE-2014-BLG-1112. The event occurred on a star that is located toward the Galactic bulge field. The equatorial coordinates of the lensed star (source) are (R.A., decl. $)_{\mathrm{J} 2000}=\left(18^{\mathrm{h}} 08^{\mathrm{m}} 36^{\mathrm{s}} 31,-28^{\circ} 39^{\prime} 56^{\prime \prime} 9\right)$, which correspond to the Galactic coordinates $(l, b)=\left(2.76,-4^{\circ} .22\right)$. The lensing event was found by the Early Warning System of the Optical Gravitational Lensing Experiment (OGLE: Udalski et al. 2015), which has conducted a microlensing survey since 1992 using the $1.3 \mathrm{~m}$ telescope at Las Campanas Observatory in Chile. The event was also in the footprint of the Microlensing Observations in Astrophysics (MOA) survey, where the event was dubbed MOA-2014-BLG-368. We also note that there exist data taken by follow-up observations. However, we do not use the MOA and follow-up data other than those taken from the Salerno University Observatory because (1) the coverage by the OGLE data is dense enough and (2) the photometry quality of the MOA and follow-up data is not adequate enough to measure subtle higher-order effects. We use the Salerno data because they cover the peak region of the light curve. To be noted is that the lensing-induced brightening of the source star started before the 2014 bulge season, lasted throughout the season, and continued after the season.

Figure 1 shows the light curve of the lensing event. At first sight, the light curve appears to be that of a point-source pointlens (PSPL) event with a smooth and symmetric shape. The 
Table 1

Error Bar Correction Factors

\begin{tabular}{lrlc}
\hline \hline Data Set & $N_{\text {data }}$ & $k$ & $\sigma_{\text {min }}$ \\
\hline OGLE $(I)$ & 1557 & 1.534 & 0.002 \\
OGLE $(V)$ & 101 & 0.955 & 0.010 \\
Salerno $(I)$ & 60 & 1.23 & 0.010 \\
\hline
\end{tabular}

light curve of a PSPL event is described by

$$
F=A F_{s}+F_{b} ; \quad A=\frac{u^{2}+2}{u \sqrt{u^{2}+4}},
$$

where $u=\left\{\left[\left(t-t_{0}\right) / t_{\mathrm{E}}\right]^{2}+u_{0}^{2}\right\}^{1 / 2}$ represents the lens-source separation normalized to the angular Einstein radius $\theta_{\mathrm{E}}, u_{0}$ is the separation at the moment of the closest lens-source approach $t_{0}, t_{\mathrm{E}}$ is the Einstein timescale, and $F_{s}$ and $F_{b}$ represent the fluxes from the source and blended light, respectively. A close look at the light curve, however, shows a smooth deviation from the PSPL model as shown in the residual presented in the middle panel of Figure 1. We note that the deviation persisted throughout the event.

In our analysis, we use data acquired from 6-year OGLE observations conducted during the 2010-2015 seasons, plus the Salerno data obtained from follow-up observations near the peak of the event. We include OGLE data taken before (2010-2013 seasons) and after the lensing magnification (2015 season) for the secure measurement of the baseline flux. The OGLE data are composed of two sets taken in the $I$ and $V$ bands. The $I$-band data set is composed of 1557 data points. The $V$-band data, which are composed of 101 data points, are used mainly to constrain the source star. Photometry of the OGLE data was conducted using the OGLE pipeline (Udalski 2003) that is customized based on the difference imaging analysis (Alard \& Lupton 1998; Woźniak 2000). The Salerno data are reduced by a PSF-fitting pipeline developed locally.

Error bars estimated by the pipeline, $\sigma_{0}$, are readjusted following the usual procedure described in Yee et al. (2012), i.e.,

$$
\sigma=k\left(\sigma_{0}^{2}+\sigma_{\min }^{2}\right)^{1 / 2},
$$

where the factor $\sigma_{\min }$ is used to make the error bars consistent with the scatter of data and the other factor $k$ is used to make $\chi^{2} /$ dof $=1$. In Table 1 , we list the error bar correction factors $k$ and $\sigma_{\min }$ along with the number of data points, $N_{\text {data }}$.

\section{Analysis}

Knowing the deviation of the light curve from the PSPL form, we test various interpretations of the deviation. For this, we consider effects that are known to cause deviations in lensing light curves.

We start with models under the assumption that the lens is a single mass. We first check finite-source effects (FSPL model), which occur when the source passes over or approaches very close to the lens. From FSPL modeling, it is found that the deviation cannot be attributed to the finite-source effect because the deviation lasted $\gtrsim 120$ days, while the effect of the finite source is confined to a short time range. We therefore check two known causes of long-term deviations. The first one is the microlens-parallax effect. Light curve deviation by the parallax effect is caused by the deviation of the relative lens-source motion from a rectilinear trajectory due to the orbital motion of the Earth around the Sun (Gould \& Loeb 1992). In addition to the single-lensing parameters, i.e., $t_{0}, u_{0}$, and $t_{\mathrm{E}}$, consideration of the parallax effect requires the inclusion of two additional parameters $\pi_{\mathrm{E}, N}$ and $\pi_{\mathrm{E}, E}$, which denote the north and east components of the microlens-parallax vector $\pi_{\mathrm{E}}$ projected on the sky in the north and east equatorial coordinates, respectively. We also check the possibility that the source is composed of two stars: the "binary-source point-lens" (BSPL) model (Griest \& Hu 1992). Considering the source binarity requires the inclusion of three additional parameters $t_{0,2}, u_{0,2}$, and $q_{f}=F_{s 2} / F_{s 1}$, which represent the time and impact parameter of the closest lens approach to the source companion, and the flux ratio between the source stars, respectively (Han \& Jeong 1998). We find that neither of these interpretations provide a model that can explain the deviation.

We then check the possibility of the lens binarity. Under the assumption of a rectilinear relative lens-source motion, the lensing light curve of a binary-lens event is described by seven principal parameters. Three of these parameters are the same as those of a single-lens event, i.e., $t_{0}, u_{0}$, and $t_{\mathrm{E}}$. Two other parameters are needed to describe the binary lens, including the projected separation, $s$, and the mass ratio, $q$, between the lens components. The separation is normalized to $\theta_{\mathrm{E}}$. The source trajectory angle, $\alpha$, which is defined as the angle between the source trajectory and the binary-lens axis, is needed to specify the source trajectory with respect to the lens. Finally, the normalized source radius $\rho$, which is defined as the ratio of the angular source radius $\theta_{*}$ to the angular Einstein radius, i.e., $\rho=\theta_{*} / \theta_{\mathrm{E}}$, is needed to describe the deviation in the causticcrossing part of the lensing light curve caused by finite-source effects. For a single lens, the reference position on the lens plane is that of the lens itself, but for a binary lens, for which there exist two lens components, one needs to define a reference position. We use the barycenter of the binary lens as a reference position. Although this is not apparent, the light curve may be associated with caustics, during which the light curve was affected by finite-source effects. We compute finite-source magnifications using the numerical ray-shooting method. In this process, we consider the limb-darkening variation of the source star surface with the model of the surface brightness profile $S \propto 1-\Gamma(1-3 \cos \phi / 2)$, where $\Gamma$ is the limbdarkening coefficient and $\phi$ is the angle between the line of sight toward the source center and the normal to the source surface. The adopted limb-darkening coefficients, $\Gamma_{I}=0.53$ and $\Gamma_{V}=0.73$ for the $I$ and $V$ band data sets, respectively, are chosen from Claret (2000), considering the source type that is determined based on the dereddened color $(V-I)_{0}$ and brightness $I_{0}$. See Section 4 for the detailed procedure of $(V-I)_{0}$ and $I_{0}$ determinations.

Due to the multiplicity of the lensing parameters and the resulting diversity of light curves, it is known that light curves of binary-lensing events are subject to various types of degeneracy where different combinations of the lensing parameters result in similar lensing light curves. In the preliminary search, therefore, we conduct a grid search for lensing solutions not only to find the lensing solution but also to check the existence of degenerate solutions. The grid search is conducted in the space of $(s, q, \alpha)$ parameters for which lensing magnifications vary sensitively to the small changes of these parameters. The ranges of $s$ and $q$ parameters are 


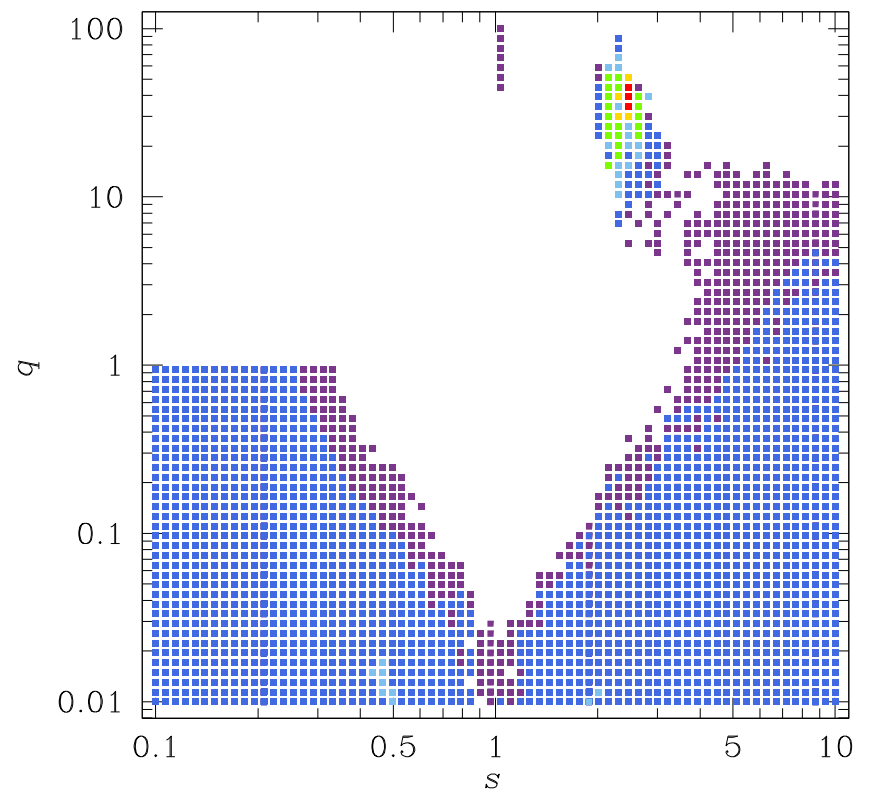

Figure 2. $\Delta \chi^{2}$ distribution in the $s-q$ parameter space obtained from the preliminary grid search for lensing solutions. The color-coding denotes points in the MCMC chain with $1 n \sigma$ (red), $2 n \sigma$ (yellow), $3 n \sigma$ (green), $4 n \sigma$ (cyan), $5 n \sigma$ (blue), and $6 n \sigma$ (purple), where $n=15$.

$-1<\log s<1$ and $-4<\log q<2$, respectively. The magnification variation to the changes of the other lensing parameters is smooth, thus we search for these parameters by minimizing $\chi^{2}$ using a downhill approach. For the downhill approach, we use the Markov Chain Monte Carlo (MCMC) method.

From the preliminary grid search, we find a unique solution of lensing parameters describing part of the observed light curve. Figure 2 shows the location of the solution on the $\Delta \chi^{2}$ distribution in the $s-q$ parameter space obtained from the preliminary grid search. The estimated values of the binary separation and mass ratio are $s \sim 2.4$ and $q \sim 40$, respectively. The value of $s$ indicates that the projected separation between the lens components is $\sim 2.4$ times greater than the angular Einstein radius. The fact that the mass ratio is greater than unity indicates that the source approached closer to the lower-mass lens component. The large mass ratio of $q \sim 40$ indicates that the companion is a low-mass object.

Since the event lasted throughout the whole bulge season, the light curve of the event might be affected by long-term higherorder effects, such as the effects of the Earth's orbital motion and the orbital motion of the lens itself (Albrow et al. 2000; Shin et al. 2012; Park et al. 2013). We therefore conduct additional modeling considering these higher-order effects. In the "parallax" and "orbit" models, we separately consider the parallax effect and the lens-orbital motion, respectively. In the "orbit +parallax" model, we simultaneously consider both higher-order effects. The parameters describing the parallax effect, $\pi_{\mathrm{E}, N}$ and $\pi_{\mathrm{E}, E}$, are the same as those defined for single-lens events. To the first-order approximation, the lens-orbital effect is described by two parameters, $d s / d t$ and $d \alpha / d t$, which represent the change rates of the binary separation and the source trajectory angle, respectively. When the parallax effect is considered, we check the degeneracy in the parallax parameters between the pair of models with $u_{0}<0$ and $u_{0}>0$. This so-called "ecliptic degeneracy" is caused by the mirror symmetry of the source trajectory with respect to the binary axis (Skowron et al. 2011).
Table 2

Comparison of Models

\begin{tabular}{clr}
\hline \hline & Model & $\chi^{2}$ \\
\hline PSPL & (Parallax) & 4371.2 \\
FSPL & (Parallax) & 4283.5 \\
BSPL & (Parallax) & 3729.8 \\
Binary & (Standard) & 1771.8 \\
$\ldots$ & (Parallax,$\left.u_{0}<0\right)$ & 1565.5 \\
$\ldots$ & (Parallax,$\left.u_{0}>0\right)$ & 1563.3 \\
$\ldots$ & (Orbit) & 1535.4 \\
$\ldots$ & (Orbit + parallax, $\left.u_{0}<0\right)$ & 1532.5 \\
$\ldots$ & (Orbit + parallax,$\left.u_{0}>0\right)$ & 1533.1 \\
\hline
\end{tabular}

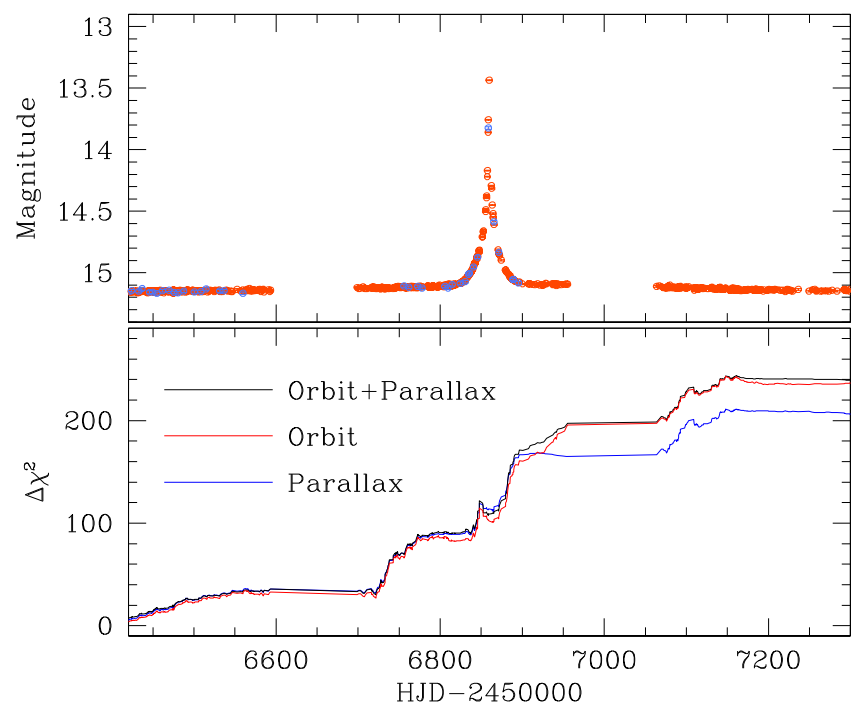

Figure 3. Cumulative $\Delta \chi^{2}$ distributions of the models with higher-order effects with respect to the model without considering the higher-order effects. The event light curve in the upper panel is presented to show the region of $\chi^{2}$ improvement.

The parameters of the two degenerate solutions are in the relation $\left(u_{0}, \alpha, \pi_{\mathrm{E}, N}\right) \leftrightarrow-\left(u_{0}, \alpha, \pi_{\mathrm{E}, N}\right)$.

In Table 2, we compare the goodness of fits of the tested models. For comparison, we also present the $\chi^{2}$ values of the PSPL, FSPL, and BSPL models. From the comparison, we find that the $\chi^{2}$ difference between the single-mass (PSPL, FSPL, and BSPL) and binary-lens models is $\Delta \chi^{2}>2000$, indicating that the event was produced by a binary object. From a comparison of the $\chi^{2}$ values of the individual binary-lens models, we find that separate considerations of the parallax and lens-orbital effects improve the fit by $\sim 208$ and 236, respectively, with respect to the model based on the principal binary-lensing parameters ("standard model"). We also find that simultaneous consideration of both higher-order effects improves the fit by $\sim 239$. Although the $\chi^{2}$ difference between the "orbit" and "orbit+parallax" models is minor, i.e., $\sim 3$, it is found that the microlens-parallax parameters with and without considering the lens-orbital effects are slightly different and thus we judge that both higher-order effects are important for a precise description of the observed lensing light curve. In Figure 3, we present a cumulative distribution of the $\Delta \chi^{2}$ of the models, considering higher-order effects with respect to the model without considering the higher-order effects. As expected from the long-term microlens-parallax and lensorbital effects, the improvement of the fit occurs throughout the event. In Figure 4, we present the distributions of $\Delta \chi^{2}$ in the 


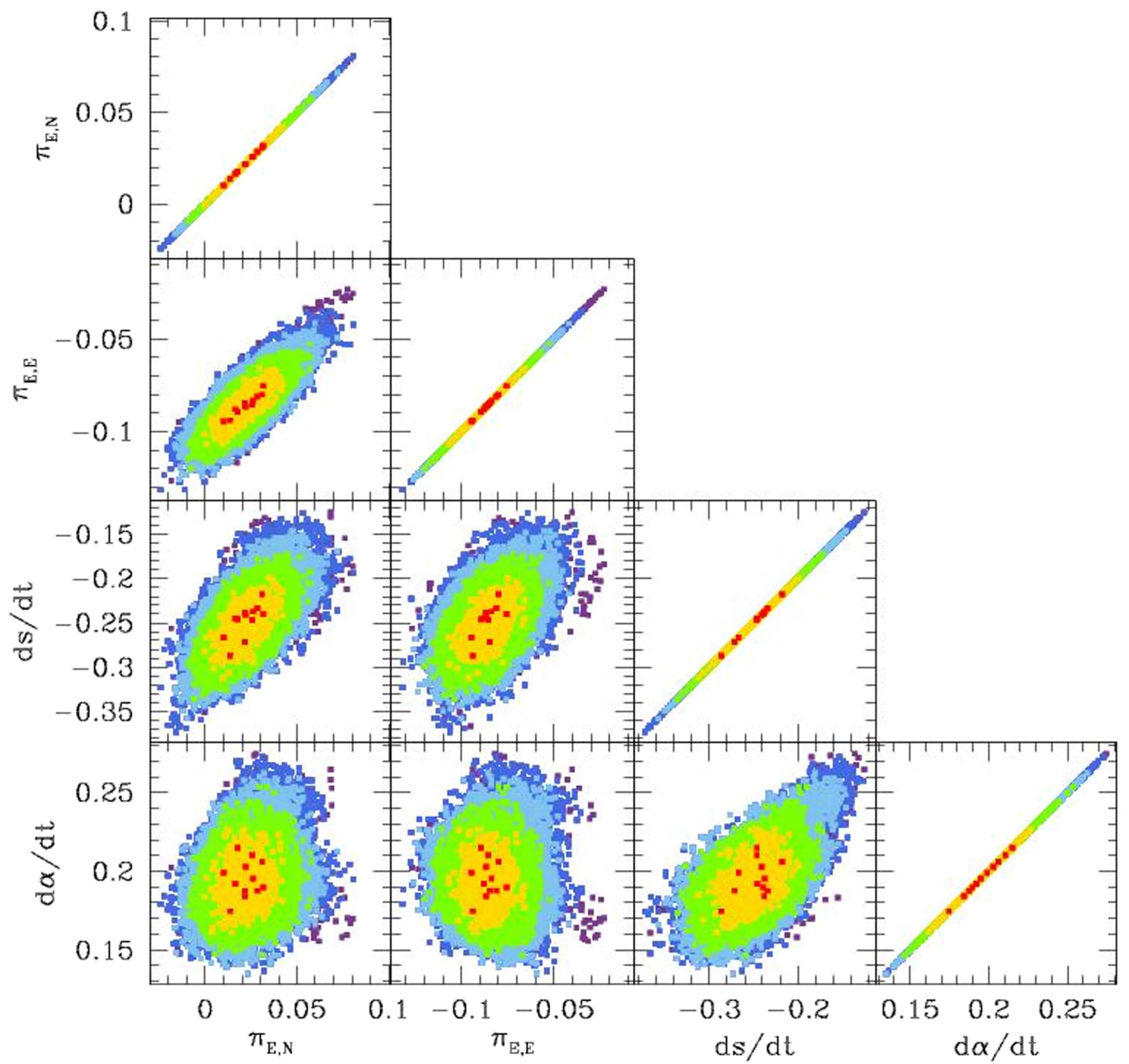

Figure 4. $\Delta \chi^{2}$ Distributions of higher-order lensing parameters: $\pi_{\mathrm{E}, N}, \pi_{\mathrm{E}, E}, d s / d t$, and $d \alpha / d t$. The color-coding denotes points in the MCMC chain with $1 \sigma$ (red), $2 \sigma$ (yellow), $3 \sigma$ (green), $4 \sigma$ (cyan), and $5 \sigma$ (blue) of the best-fit value. We note that the distributions are for the orbit + parallax model with $u_{0}<0$.

parameter space of the higher-order lensing parameters. We note that although the measured microlens-parallax value $\pi_{\mathrm{N}}=\left(\pi_{\mathrm{E}, N}^{2}+\pi_{\mathrm{E}, E}^{2}\right)^{1 / 2}=0.092$ is small, it is measured with a significant confidence level.

In Table 3, we present the lensing parameters of the best-fit model, i.e., "orbit+parallax" binary-lens model. Since the ecliptic degeneracy is very severe (with $\Delta \chi^{2}=0.6$ ), we present both the $u_{0}<0$ and $u_{0}>0$ solutions. In Figure 5, we also present the lens-system geometry, which shows the source trajectory (curve with an arrow) with respect to the lens components (marked by blue dots) and the caustic (red cuspy closed curves). We note that the upper and lower panels correspond to the $u_{0}<0$ and $u_{0}>0$ solutions, respectively. It is found that the lens is a wide binary for which two sets of caustics form near the individual lens components. The event was produced by the source trajectory, which passed the tip of the caustic located close to the lower-mass lens component, $M_{2}$. Since the source trajectory almost vertically passed the binary axis $\left(\alpha \sim 80^{\circ}\right)$, the light curve appears to be symmetric with respect to the peak that corresponds to the time of the causticcrossing. In Figure 1, we present the best-fit model light curve, i.e., the "orbit + parallax" model with $u_{0}<0$, superposed onto
Table 3

Best-fit Lensing Parameters

\begin{tabular}{lcc}
\hline \hline Parameters & $u_{0}>0$ & $u_{0}<0$ \\
\hline$t_{0}\left(\mathrm{HJD}^{\prime}\right)$ & $6892.200 \pm 0.994$ & $6890.985 \pm 0.174$ \\
$u_{0}$ & $-1.848 \pm 0.011$ & $1.861 \pm 0.001$ \\
$t_{\mathrm{E}}($ days $)$ & $107.44 \pm 1.10$ & $106.37 \pm 0.85$ \\
$s$ & $2.43 \pm 0.01$ & $2.42 \pm 0.01$ \\
$q$ & $35.37 \pm 1.56$ & $47.38 \pm 3.35$ \\
$\alpha(\mathrm{rad})$ & $1.404 \pm 0.008$ & $-1.411 \pm 0.001$ \\
$\rho\left(10^{-3}\right)$ & $1.14 \pm 0.03$ & $1.01 \pm 0.04$ \\
$\pi_{\mathrm{E}, N}$ & $0.02 \pm 0.01$ & $-0.01 \pm 0.01$ \\
$\pi_{\mathrm{E}, E}$ & $-0.09 \pm 0.01$ & $-0.08 \pm 0.01$ \\
$d s / d t\left(\mathrm{yr}^{-1}\right)$ & $-0.25 \pm 0.03$ & $-0.29 \pm 0.03$ \\
$d \alpha / d t\left(\mathrm{yr}{ }^{-1}\right)$ & $0.22 \pm 0.02$ & $-0.40 \pm 0.05$ \\
$\left(F_{s} / F_{b}\right)_{\text {OGLE }, I}$ & $13.49 / 0.26$ & $13.69 / 0.06$ \\
\hline
\end{tabular}

Note. HJD' $=$ HJD-2450000.

the data points. In the bottom panel, we also present the residual of the model. One finds that the residual from the FSPL model has gone with the introduction of the lens binarity. 


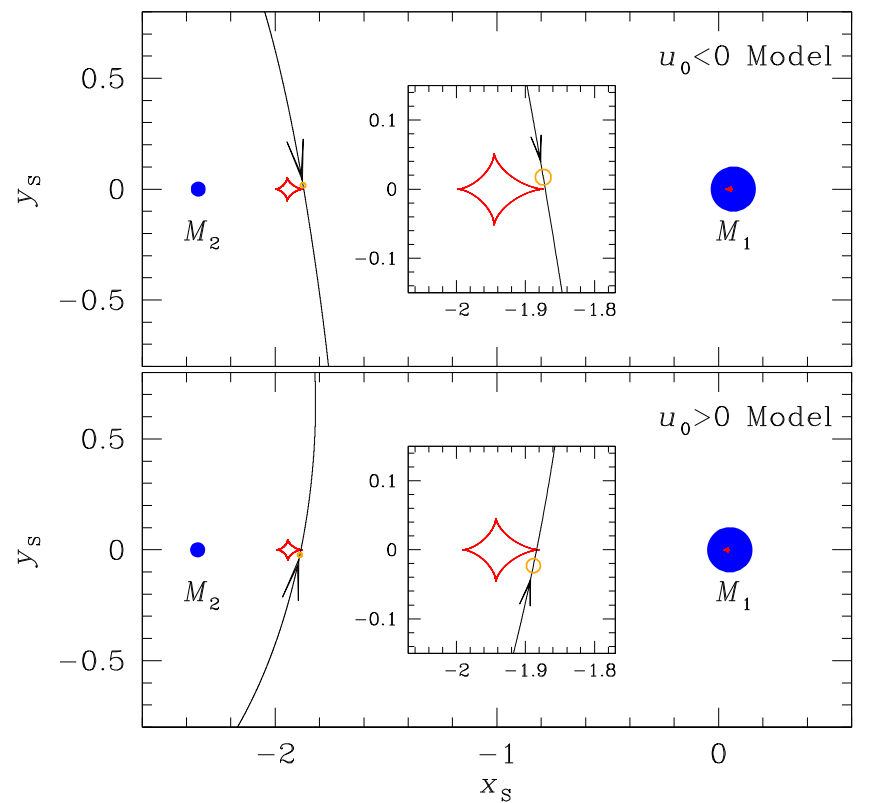

Figure 5. Geometry of the lens system. The curve with an arrow is the source trajectory and the blue dots represent the locations of the binary-lens components, where $M_{1}$ and $M_{2}$ denote the heavier and lighter mass components. The cuspy close curve represents the caustic. The inset shows the enlarged view of the caustic that is located closer to the lower-mass lens component. All lengths are normalized to the angular Einstein ring radius corresponding to the total mass of the binary lens, and the coordinates are centered at the barycenter of the binary lens.

We note that finite-source effects are clearly detected, although there exist none of the prominent spike causticcrossing features that commonly appear in binary-lens light curves. This can be seen in the peak region of the light curve presented in Figure 6, where we plot two model light curves with a point (dotted curve) and a finite source (solid curve). The measured values of the normalized source radius are $\rho=(1.14 \pm 0.03) \times 10^{-2}$ and $(1.01 \pm 0.03) \times 10^{-2}$ for the $u_{0}<0$ and $u_{0}>0$ solutions, respectively. In the inset of Figure 5, we mark the source near the caustic as a circle where the size of the circle is scaled to the caustic size. It shows that the source crossed the tip of the caustic. We note that finite-source effects could also have been detected through specific features of polarization curves, if a polarization observation of the event were conducted (Ingrosso et al. 2012, 2014).

\section{Physical Parameters}

While subtle modulation of the lensing light curve allows us to measure the microlens parallax $\pi_{\mathrm{E}}$, detections of the finitesource effect enable us to measure the angular Einstein radius $\theta_{\mathrm{E}}$, which is the other ingredient for the lens mass determination. The angular Einstein radius is related to the normalized source radius $\rho$ and the angular source radius $\theta_{*}$ by $\theta_{\mathrm{E}}=\theta_{*} / \rho$, and thus one needs to estimate $\theta_{*}$ for the $\theta_{\mathrm{E}}$ measurement. We estimate the angular source radius from the dereddened color and brightness following the method of Yoo et al. (2004), using the centroid of the bulge giant clump (GC) as a standard candle. For this method, the dereddened color and magnitude of the source stars are determined by

$$
(V-I, I)_{0}=(V-I, I)_{0, \mathrm{GC}}+\Delta(V-I, I),
$$

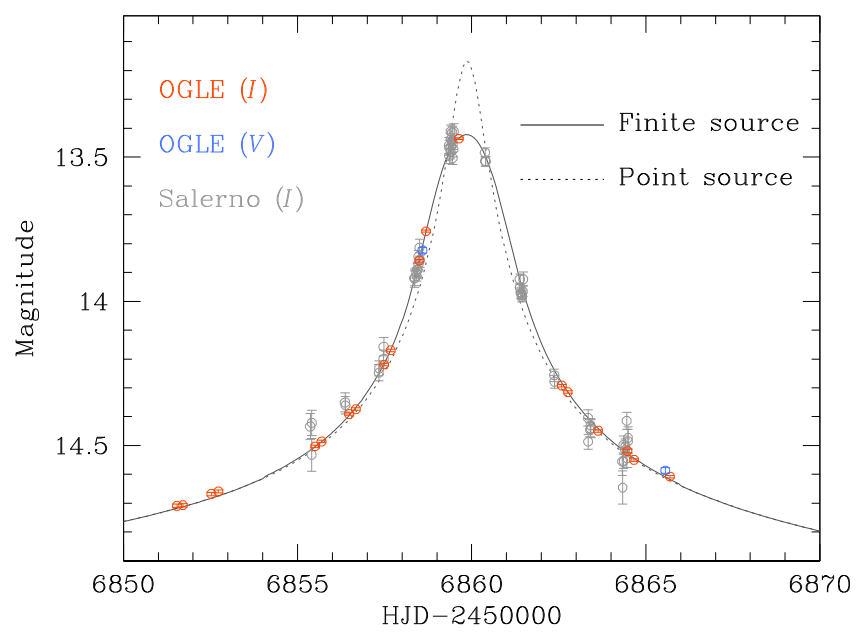

Figure 6. Peak region of the light curve. The dotted and solid curves represent the model light curves with a point and a finite source, respectively.

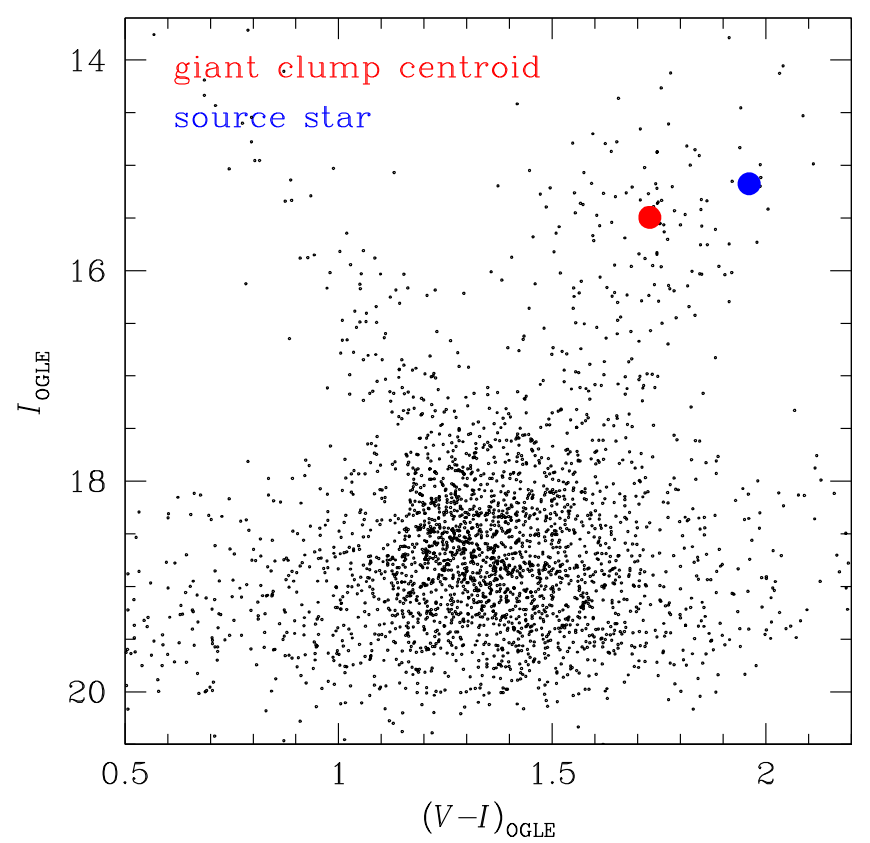

Figure 7. Source location with respect to that of the centroid of the giant clump in the color-magnitude diagram.

where $(V-I, I)_{0, \mathrm{GC}}=(1.06,14.29)$ are the intrinsic color and magnitude of the GC centroid (Bensby et al. 2011; Nataf et al. $2013)$ and $\Delta(V-I, I)$ are offsets of the color and magnitude of the source star from those of the GC centroid measured in the instrumental (uncalibrated) color-magnitude diagram. In Figure 7, we mark the locations of the source and centroid of the GC in the instrumental color-magnitude diagram of stars in the neighboring region around the source star. We find that the dereddened color and magnitude of the source star are $(V-I, I)_{0}=$ $(1.29,13.98)$, indicating that the source is a K-type giant. We then estimate the angular source radius by first converting $V-I$ into $V-K$ using the color-color relation (Bessell \& Brett 1988) and then estimate $\theta_{*}$ using the relation between $V-K$ and the surface brightness (Kervella et al. 2004).

In Table 4, we present the estimated angular source radius and Einstein radius. Also presented are the relative lens-source proper motions measured in the geocentric and heliocentric 
Table 4

Source Parameters

\begin{tabular}{lcc}
\hline \hline Quantity & $u_{0}<0$ & $u_{0}>0$ \\
\hline$\theta_{*}($ uas $)$ & $9.30 \pm 1.35$ & $9.37 \pm 1.36$ \\
$\theta_{\mathrm{E}}($ mas $)$ & $0.82 \pm 0.12$ & $0.93 \pm 0.14$ \\
$\mu_{\text {geo }}\left(\right.$ mas yr $\left.^{-1}\right)$ & $2.78 \pm 0.41$ & $3.17 \pm 0.48$ \\
$\mu_{\text {helio }}\left(\right.$ mas yr $\left.^{-1}\right)$ & $2.52 \pm 0.38$ & $2.93 \pm 0.44$ \\
\hline
\end{tabular}

frames. The geocentric proper motion is estimated by

$$
\mu_{\mathrm{geo}}=\frac{\theta_{\mathrm{E}}}{t_{\mathrm{E}}}
$$

The heliocentric proper motion is measured by

$$
\boldsymbol{\mu}_{\text {helio }}=\boldsymbol{\mu}_{\text {geo }}+v_{\oplus, \perp} \frac{\pi_{\text {rel }}}{\mathrm{au}},
$$

where $\boldsymbol{\mu}_{\mathrm{geo}}=\left[\mu_{\mathrm{geo}}\left(\pi_{\mathrm{E}, N} / \pi_{\mathrm{E}}\right), \mu_{\mathrm{geo}}\left(\pi_{\mathrm{E}, E} / \pi_{\mathrm{E}}\right)\right], \pi_{\mathrm{rel}}$ is the relative lens-source parallax, and $v_{\oplus, \perp}$ is the velocity of the Earth projected on the sky at $t_{0}$. We note that the direction of $\boldsymbol{\mu}_{\text {helio }}$ is the same as that of the microlens-parallax vector $\pi_{\mathrm{E}}$.

By measuring both the microlens parallax and the angular Einstein radius, we can determine the mass of the lens using the relation in Equation (1). One can also determine the distance to the lens using the relation (Gould 2000)

$$
D_{\mathrm{L}}=\frac{\mathrm{au}}{\pi_{\mathrm{E}} \theta_{\mathrm{E}}+\pi_{\mathrm{S}}},
$$

where $\pi_{\mathrm{S}}=\mathrm{au} / D_{\mathrm{S}}$ is the parallax of the source star and $D_{\mathrm{S}}$ is the distance to the source star. In Table 5, we present the determined physical parameters. The masses of the lens components are estimated by $M_{1}=M /(1+q)$ and $M_{2}=M q /(1+q)$, where $M=M_{1}+M_{2}$. The projected separation between the lens components is estimated by $a_{\perp}=s D_{\mathrm{L}} \theta_{\mathrm{E}}$. Also presented is the projected kinetic-topotential-energy ratio, which is computed by

$$
\left(\frac{\mathrm{KE}}{\mathrm{PE}}\right)_{\perp}=\frac{\left(r_{\perp} / \mathrm{au}\right)^{3}}{8 \pi^{2}\left(M / M_{\odot}\right)}\left[\left(\frac{1}{s} \frac{d s}{d t}\right)^{2}+\left(\frac{d \alpha}{d t}\right)^{2}\right] .
$$

In order for the binary lens to be a bound system, the ratio must satisfy the condition $(\mathrm{KE} / \mathrm{PE})_{\perp} \leqslant \mathrm{KE} / \mathrm{PE} \leqslant 1$, where $\mathrm{KE} / \mathrm{PE}$ is the intrinsic energy ratio. It is found that the energy ratio for the $u_{0}>0$ model is greater than unity, indicating that the solution results in unphysical parameters. This leaves the $u_{0}<0$ model as the only viable solution.

According to the solution, the lens is composed of a solar-type primary star with a mass of $M_{1}=1.07 \pm 0.28 M_{\odot}$ and a brown dwarf companion with a mass of $M_{2}=(3.03 \pm 0.78) \times$ $10^{-2} M_{\odot}$. The companion has a mass less than that of the hydrogen-burning limit of $\sim 0.08 M_{\odot}$ (Hayashi \& Nakano 1963; Nakano 2014). The projected separation between the lens components is $a_{\perp}=9.63 \pm 1.33 \mathrm{au}$. The lens is located at a distance $D_{\mathrm{L}}=4.84 \pm 0.67 \mathrm{kpc}$.

\section{Discussion}

Before 2014, nearly all microlens mass measurements were based on the "annual parallax," which is determined from the modulation of a lensing light curve caused by the annual orbital motion of the Earth. A microlens parallax can also be measured
Table 5

Physical Lens Parameters

\begin{tabular}{lcr}
\hline \hline \multirow{2}{*}{ Parameter } & \multicolumn{2}{c}{ Model } \\
\cline { 2 - 3 } & $u_{0}>0$ & $u_{0}<0$ \\
\hline$M_{1}\left(M_{\odot}\right)$ & $1.07 \pm 0.28$ & $1.40 \pm 0.40$ \\
$M_{2}\left(10^{-2} M_{\odot}\right)$ & $3.03 \pm 0.78$ & $2.95 \pm 0.84$ \\
$D_{\mathrm{L}}(\mathrm{kpc})$ & $4.84 \pm 0.67$ & $4.87 \pm 0.71$ \\
$a_{\perp}(\mathrm{au})$ & $9.63 \pm 1.33$ & $10.90 \pm 1.58$ \\
$(\mathrm{KE} / \mathrm{PE})_{\perp}$ & 0.58 & 2.00 \\
\hline
\end{tabular}

by conducting simultaneous observations of lensing events from a ground-based observatory and from a satellite in solar orbit (Refsdal 1966; Gould 1994). Since 2014, a space-based microlensing campaign using the Spitzer telescope, which is in solar orbit with a projected separation toward the bulge of $d_{\text {proj }} \sim 1$ au, has been conducted (Calchi Novati et al. 2015). By successfully measuring microlens parallaxes of various types of lensing events, e.g., Udalski et al. (2015), Street et al. (2016), Yee et al. (2015), Shvartzvald et al. (2016), Zhu et al. (2015), Bozza et al. (2016), and Han et al. (2016), the campaign demonstrated that space-based parallaxes can be routinely measured for general lensing events. In the 2016 season, a new microlensing survey was conducted using the Kepler satellite, which has projected separations spanning $0.07 \mathrm{au}<d_{\text {proj }}<0.81$ au, over the course of the 2016 season (Henderson et al. 2016). The data collected from the campaign, dubbed K2's Campaign 9 (K2C9), are being processed and microlens parallaxes of numerous events are expected to be measured. Furthermore, the WFIRST space telescope, which is set to launch in the mid-2020s, will be able to measure spacebased microlens parallaxes for an increased number of lensing events.

Binary events will be important targets for detections of brown dwarfs in current and future space-based lensing observations. For binary-lens events, angular Einstein radii are routinely measurable and thus additional measurements of space-based microlens parallaxes will enable measurements of lens masses. Furthermore, while the microlens-parallax measurement for a single-mass event suffers from a well-known twofold degeneracy (Gould 1994), the microlens parallax of a binary-lens event is, in general, uniquely determined (Han et al. 2017). The ability to routinely measure the masses of binary lenses, and thus the number of microlensing brown dwarfs detected through the channel of binary-lens events, is expected to be greatly improved.

\section{Conclusion}

We reported a microlensing brown dwarf discovered from the analysis of the gravitational binary-lens event OGLE-2014BLG-1112. We identified the brown-dwarf nature of the lens companion by measuring the lens mass from the detections of both microlens-parallax and finite-source effects. We found that the companion has a mass of $(3.03 \pm 0.78) \times 10^{-3} M_{\odot}$ and it is orbiting a solar-type primary star with a mass of $1.07 \pm 0.28 M_{\odot}$. The estimated projected separation between the lens components was $9.63 \pm 1.33$ au and the distance to the lens was $4.84 \pm 0.67 \mathrm{kpc}$. We discussed the usefulness of space-based microlensing observations for detecting brown dwarfs through the channel of binary-lens events. 
Work by C. Han was supported by the grant 2017R1A4A1015178 of the National Research Foundation of Korea. The OGLE project has received funding from the National Science Centre, Poland, grant MAESTRO 2014/14/ A/ST9/00121 to A. Udalski. The OGLE Team thanks professors M. Kubiak and G. Pietrzyński, former members of the OGLE team, for their contribution to the collection of the OGLE photometric data over the past years. We acknowledge the high-speed internet service (KREONET) provided by the Korea Institute of Science and Technology Information (KISTI).

\section{References}

Alard, C., \& Lupton, R. H. 1998, ApJ, 503, 325

Albrow, M. D., Beaulieu, J.-P., Caldwell, J. A. R., et al. 2000, ApJ, 534, 894

Bensby, T., Adén, D., Meléndez, J., et al. 2011, A\&A, 533, 134

Bessell, M. S., \& Brett, J. M. 1988, PASP, 100, 1134

Bozza, V., Shvartzvald, Y., Udalski, A., et al. 2016, ApJ, 820, 79

Calchi Novati, S., Gould, A., Udalski, A., et al. 2015, ApJ, 804, 20

Claret, A. 2000, A\&A, 363, 1081

Gaudi, B. S. 2003, in Proc. IAU Symp. 211, Brown Dwarfs, ed. E. Martin (San Francisco, CA: ASP), 305

Gould, A. 1992, ApJ, 392, 442

Gould, A. 1994, ApJL, 421, L75

Gould, A. 2000, ApJ, 542, 785

Gould, A., \& Loeb, A. 1992, ApJ, 396, 104

Griest, K., \& Hu, W. 1992, ApJ, 397, 36
Han, C., \& Jeong, Y. 1998, MNRAS, 301, 231

Han, C., Jung, Y. K., Udalski, A., et al. 2016, ApJ, 822, 75

Han, C., Udalski, A., Gould, A., et al. 2017, ApJ, 834, 82

Hayashi, C., \& Nakano, T. 1963, PThPh, 30, 460

Henderson, C. B., Poleski, R., Penny, M., et al. 2016, PASP, 128, 124401

Ingrosso, G., Calchi Novati, S., de Paolis, F., et al. 2009, MNRAS, 399, 219

Ingrosso, G., Calchi Novati, S., De Paolis, F., et al. 2011, GReGr, 43, 1047

Ingrosso, G., Calchi Novati, S., De Paolis, F., et al. 2012, MNRAS, 426, 1496

Ingrosso, G., De Paolis, F., Nucita, A. A., et al. 2014, PhyS, 89, 84001

Kervella, P., Thévenin, F., Di Folco, E., \& Ségransan, D. 2004, A\&A, 426, 297

Mao, S., \& Paczyński, B. 1991, ApJL, 374, L37

Nakano, T. 2014, Astrophysics and Space Science Library, Vol. 401 (Berlin: Springer), 5

Nataf, D. M., Gould, A., Fouqué, P., et al. 2013, ApJ, 769, 88

Park, H., Udalski, A., Han, C., et al. 2013, ApJ, 778, 134

Refsdal, S. 1966, MNRAS, 134, 315

Shin, I.-G., Han, C., \& Gould, A. 2012, ApJ, 760, 116

Shvartzvald, Y., Li, Z., Udalski, A., et al. 2016, ApJ, 831, 183

Skowron, J., Udalski, A., \& Gould, A. 2011, ApJ, 738, 87

Street, R. A., Udalski, A., Calchi Novati, A., et al. 2016, ApJ, 819, 93

Udalski, A. 2003, AcA, 53, 291

Udalski, A., Szymański, M. K., \& Szymański, G. 2015, AcA, 65, 1

Udalski, A., Yee, J. C., Gould, A., et al. 2015, ApJ, 799, 237

Whitworth, A., Bate, M. R., Nordlund, A., Reipurth, B., \& Zinnecker, H. 2007, in Protostars and Planets V, ed. V. B. Reipurth et al. (Tucson, AZ: Univ. Arizona Press), 459

Woźniak, P. R. 2000, AcA, 50, 421

Yee, J. C., Shvartzvald, Y., Gal-Yam, A., et al. 2012, ApJ, 755, 102

Yee, J. C., Udalski, A., Calchi Novati, S., et al. 2015, ApJ, 802, 76

Yoo, J., DePoy, D. L., Gal-Yam, A., et al. 2004, ApJ, 603, 139

Zhu, Wei., Udalski, A., Gould, A., et al. 2015, ApJ, 805, 8 